\title{
Polarization-Independent and Angle-Insensitive Metamaterial Absorber Using 90-Degree-Rotated Split-Ring Resonators
}

\author{
Jia-Qi Feng, ${ }^{1}$ Wei-Dong Hu, ${ }^{1}$ Qing-Le Zhang, ${ }^{1}$ Hua Zong, ${ }^{1}$ Hui Huang, ${ }^{2}$ Yu-Ting Jin, \\ Yu-Ming Wu, ${ }^{1}$ Zhan Xu, ${ }^{1}$ Xin Lv, ${ }^{1}$ and Li-Ming Si ${ }^{1}$ \\ ${ }^{1}$ Beijing Key Laboratory of Millimeter Wave and Terahertz Technology, School of Information and Electronics, \\ Beijing Institute of Technology, Beijing 100081, China \\ ${ }^{2}$ Microwave Parameters Laboratory, Division of Electronics and Information Technology, National Institute of Metrology, \\ Beijing 100013, China \\ Correspondence should be addressed to Li-Ming Si; lms@bit.edu.cn
}

Received 6 March 2015; Revised 24 April 2015; Accepted 24 April 2015

Academic Editor: Roman E. Noskov

Copyright (C) 2015 Jia-Qi Feng et al. This is an open access article distributed under the Creative Commons Attribution License, which permits unrestricted use, distribution, and reproduction in any medium, provided the original work is properly cited.

\begin{abstract}
We present the design, simulation, and measurement of a polarization-independent and angle-insensitive metamaterial absorber (MA) in X-band. Since the unit cell of the MA consists of four subwavelength split-ring resonators with 4-fold symmetric rotation, the MA is insensitive to the variation of both polarization and incident angle of the planar electromagnetic wave. The electromagnetic performances of the MA are studied by full-wave simulations based on finite-element method and the Naval Research Laboratory arch experimental measurements. The electric field distributions are numerically investigated, which confirm the polarization-insensitive property of the MA, as expected from the symmetric nature of the structure. When the incident angles vary from 0 to 45 degrees, the MA remains at full width at half maximum of $0.4 \mathrm{GHz}(0.5 \mathrm{GHz})$ with peak absorptions of $99.9 \%$ (95.2\%) at $10.27 \mathrm{GHz}(10.3 \mathrm{GHz})$ by simulations (measurements).
\end{abstract}

\section{Introduction}

Metamaterials can provide exotic and very useful electric and magnetic properties which are not readily found in nature $[1,2]$. Over the past decade, a large number of metamaterials have been demonstrated at the desired regimes of electromagnetic spectrum from microwave, terahertz $(\mathrm{THz})$, infrared to visible frequencies [3-6]. As metamaterials research continues to mature, they have inspired a vast amount of electromagnetic devices such as invisibility cloaks $[7,8]$, perfect lens [9], and novel types of circuits and antennas [10-14]. One of the most promising applications of metamaterials is the absorption of electromagnetic waves, known as metamaterial absorbers (MAs) [15-29]. MAs offer three distinct advantages-nearly perfect absorption, deeply subwavelength thickness, and tunability-compared to conventional radar absorbing materials. Potential applications of MAs include the bolometer, perfectly matched layer, and radar cross section (RCS) reduction. MA with ultrathin structure and near-unity absorption was first introduced by Landy et al. in 2008 [15]. Since then, great efforts have been concentrated on MAs to achieve broadband, multiband, polarization-independent, or angle-insensitive absorptions from microwave, $\mathrm{THz}$, to optical frequencies [16-29]. During the last few years, although some of the studies show good absorptions, experimental realization of MAs with both polarization-independent and angle-insensitive properties are still in the initial stage. In future research more unit cell structures will provide greater flexibility in MA design and practical applications.

In this study, an X-band polarization-independent and angle-insensitive MA based on 90-degree-rotated split-ring resonators (SRRs) is designed and proposed. The MA is designed and optimized by full-wave simulations using finiteelement method (FEM) based commercial software ANSYS HFSS and verified by the Naval Research Laboratory (NRL) arch experimental measurements. We will show that the proposed MA yields not only a compact structure that can 


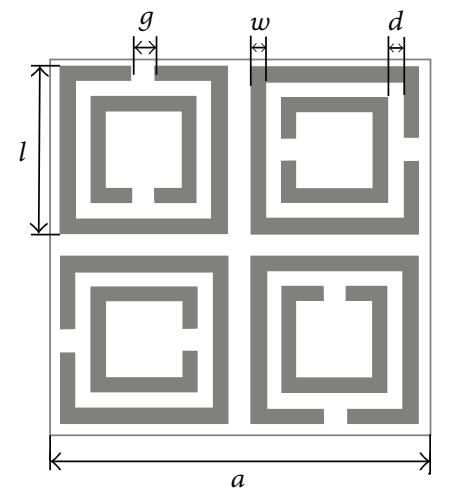

(a)

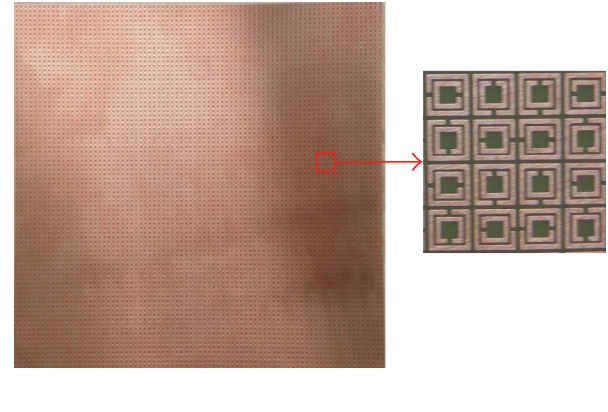

(b)

Figure 1: Schematic of MA: (a) unit cell with dimensions of $a=5, l=2.22, g=0.3, w=0.2$, and $d=0.2$. (b) Photograph of the fabricated MA with $40 \times 40$ unit cells (dimension of $200 \times 200$ ). All units are in millimeters.

be readily fabricated through standard printed circuit board (PCB) technology, but also good polarization-independent and angle-insensitive absorption characteristics from both simulation and experiment results.

\section{MA Design}

For our MA design, we chose unit cell with 4-fold rotational symmetric configuration, which leads to polarizationindependent property. Meanwhile, the MA constructed with subwavelength unit cells can offer angle-insensitivity property. Figure 1(a) shows the geometry of the unit cell of the $\mathrm{MA}$, and the fabricated prototype of the proposed MA is illustrated in Figure 1(b). The unit cell of the MA consists of four symmetric subwavelength 90-degree-rotated SRRs, and it is fabricated by etching an $18 \mu \mathrm{m}$ thick copper (conductivity $5.8 \times 10^{7} \mathrm{~S} / \mathrm{m}$ ) pattern on an FR4 (relative permittivity 4.4 and dielectric loss tangent 0.02 ) epoxy dielectric substrate with $0.8 \mathrm{~mm}$ thickness. The optimized geometrical parameters of the unit cell are listed in the caption of Figure 1(a).

The absorption $A$ of the MA can be determined by the expression [30]

$$
A=1-R-T=1-\left|S_{11}\right|^{2}-\left|S_{21}\right|^{2},
$$

where reflection $R=\left|S_{11}\right|^{2}$ and transmission $T=\left|S_{21}\right|^{2}$ are obtained from simulated and measured $S$-parameters. From this equation, reflection $R$ and transmission $T$ should be minimized to achieve maximum absorption $A$. The transmission $T$ of the proposed MA is equal to zero, $S_{21}=0$, due to the presence of the metallic ground plane in our design. Therefore, the absorption $A$ of the MA can be maximized by minimization of the reflection $R$.

To better understand the mechanism of the perfect absorption of the MA, the effective impedance of the MA can be obtained from effective medium theory [31-33]:

$$
z=\sqrt{\frac{\left(1+S_{11}^{2}\right)^{2}-S_{21}^{2}}{\left(1-S_{11}^{2}\right)^{2}-S_{21}^{2}}}=\frac{1+R}{1-R} .
$$

Combination of (1) and (2) results in the general expression of the absorption:

$$
\begin{aligned}
A= & \frac{2}{\operatorname{Re}(z)+i \operatorname{Im}(z)+1} \\
= & \frac{2[\operatorname{Re}(z)+1]}{[\operatorname{Re}(z)+1]^{2}+\operatorname{Im}(z)^{2}} \\
& -i \frac{2 \operatorname{Im}(z)}{[\operatorname{Re}(z)+1]^{2}+\operatorname{Im}(z)^{2}} .
\end{aligned}
$$

In case of perfect absorption, $A=1$, the real part of the effective impedance should match the free-space value, $\operatorname{Re}(z)=1$, and the imaginary part is supposed to be vanished, $\operatorname{Im}(z)=0$, according to (3).

\section{Simulations and Measurements}

The MA with three layers is modelled with full-wave FEM solver ANSYS HFSS. The top layer is made of 90-degreerotated SRRs in a periodic pattern. The middle layer is dielectric substrate FR4. The bottom layer is a solid metallic ground plane. The simulation is based on a MA unit cell along with Floquet ports and Master/Slave periodic boundary condition (PBC). The usage of PBC can mimic an infinite extended periodic structure.

The simulated $S_{11}$ of the MA is shown in Figure 2. A minimal reflection of near zero is observed at $10.27 \mathrm{GHz}$. By using (1) and (2), the absorption and effective impedance can be easily calculated. The effective impedance is calculated and shown in Figure 3. The inset in Figure 3 shows the zoomed view of the effective impedance. It can be observed that the real part of the effective impedance of the MA is near unity (matched to the air), $\operatorname{Re}(z) \cong 1$, and imaginary part of the MA is near zero, $\operatorname{Im}(z) \cong 0$ at $10.27 \mathrm{GHz}$, corresponding to the perfect absorption. Figure 4 shows the absorption as a function of polarization angle with step of $10^{\circ}$ at $10.27 \mathrm{GHz}$. The absorption amplitude for different polarization angles 


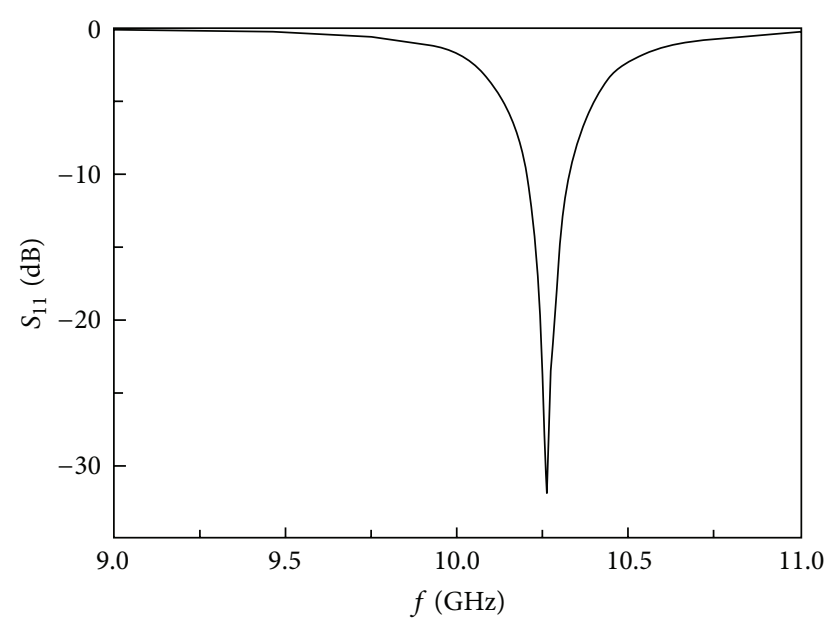

Figure 2: The simulated $S_{11}$ of the proposed MA from a normal incident planar wave.

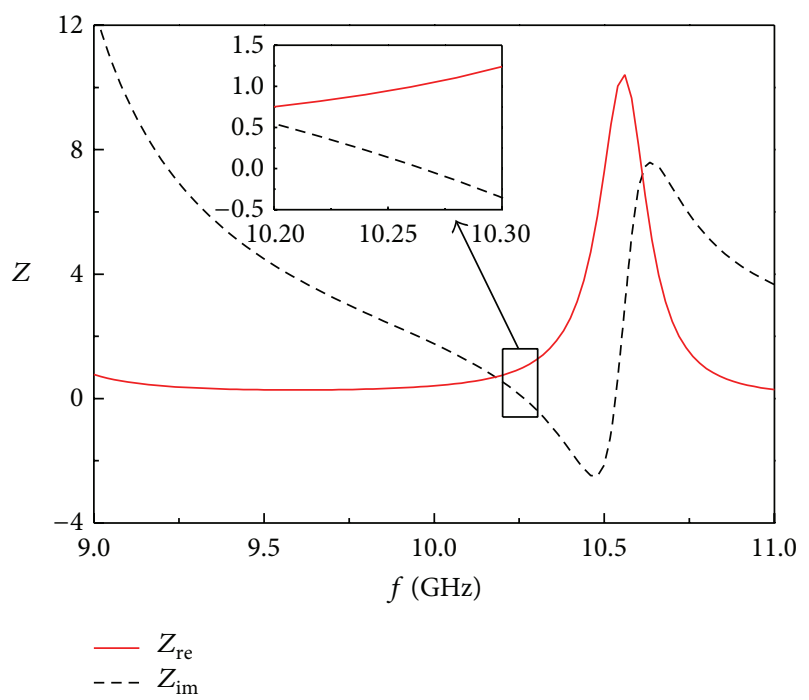

Figure 3: Calculated real and imaginary part of the effective impedance of the proposed MA.

varies by less than $1 \%$. The detailed discussion on angleinsensitive property of the proposed MA is presented in the fifth paragraph of this section.

Figure 5 shows the simulated electric field distribution at 10.27 GHz for normal incident TE and TM waves, as well as the $45^{\circ}$ condition. It is seen that the MA performs almost the same electric field distributions, except a 90-degree rotation, for normal incident TE and TM waves, as shown in Figures 5(a) and 5(b). This indicates that the symmetrical structure of the MA with the unit cell of 4-fold rotational SRRs possesses a polarization-independent performance, in accordance with our predication. In Figure 5(c), the electric field distribution for $45^{\circ}$ incident electromagnetic waves is shown at $10.27 \mathrm{GHz}$. In this condition, we discovered that all of the incident electromagnetic waves are absorbed by the four 90 -degreerotated SRRs.

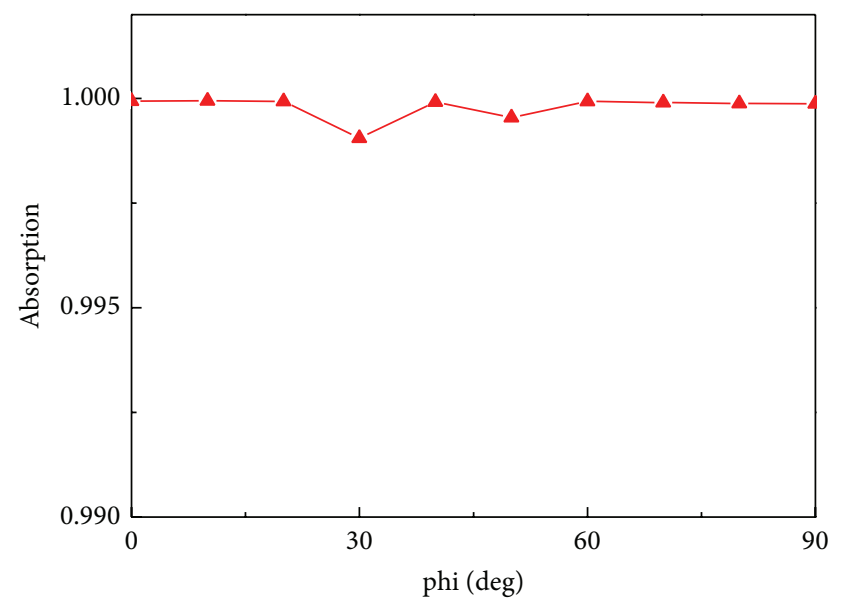

FIGURE 4: Simulated absorption as a function of polarization angle with step of $10^{\circ}$ at $10.27 \mathrm{GHz}$.

To verify the numerical results, we fabricate the MA by using PCB technique, as shown in Figure 1(b). The absorption characteristics of the fabricated MA at different incident angles are measured with the NRL arch, as shown in Figure 6. NRL arch is a reliable standard for characterizing the reflection and absorption of materials [34]. In our measurements, a vector network analyzer (Agilent N5244A) is used to measure the reflection of the proposed MA.

Figure 7 shows the simulated and measured absorption at incident angles from 0 to 45 degrees in X-band. It can be seen that when the incident angle of the electromagnetic wave is altered from 0 to 45 degrees, the absorption does not change significantly, which proves that our proposed MA possesses angle-insensitive property. It is known that a periodic structure with subwavelength dimensions of unit cell is equivalent to a homogenous and isotropic medium. The effective impedance of a homogenous and isotropic medium is stable for wide angles of incident electromagnetic waves. Therefore, the strong subwavelength dimensions of the lattice constant of unit cell $(a=5 \mathrm{~mm})$ and dielectric thickness $(0.8 \mathrm{~mm})$ of the MA make it absorb wide angles of incident electromagnetic waves. The measured results are in good agreement with numerical simulations. The small discrepancy of the peak absorption with the change of incident angle might be due to nonidealities of fabrication, such as the surface roughness of the metal pattern and nonuniformity of the dielectric substrate.

Furthermore, the full width at half maximum (FWHM) and fractional bandwidth (FBW) are very important parameters to describe the performance of the MA. Fractional bandwidth (FBW) was calculated as

$$
\mathrm{FBW}=\frac{\mathrm{FWHM}}{f_{c}},
$$

where $f_{c}$ is the center frequency of FWHM. For incident angles varying from 0 to 45 degrees, the MA remains at the FWHM of $0.4 \mathrm{GHz}$ with peak absorptions of $99.9 \%$ at $10.27 \mathrm{GHz}$ in simulations, while in experiments the MA remains at an FWHM of $0.5 \mathrm{GHz}$ with peak absorptions 


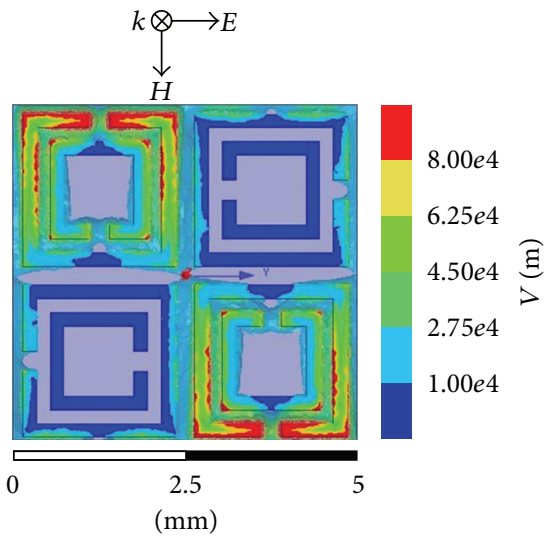

(a)

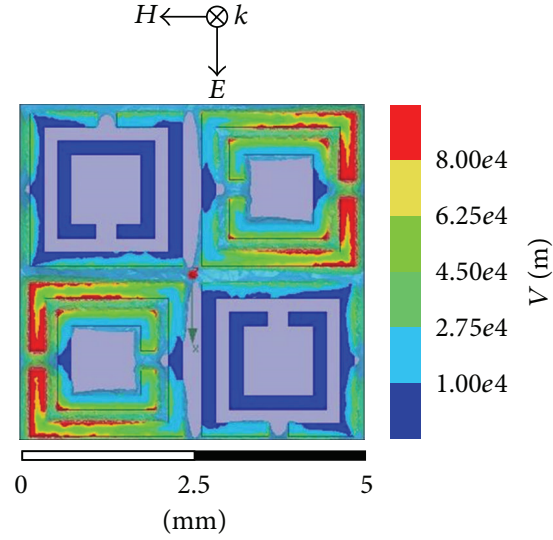

(b)

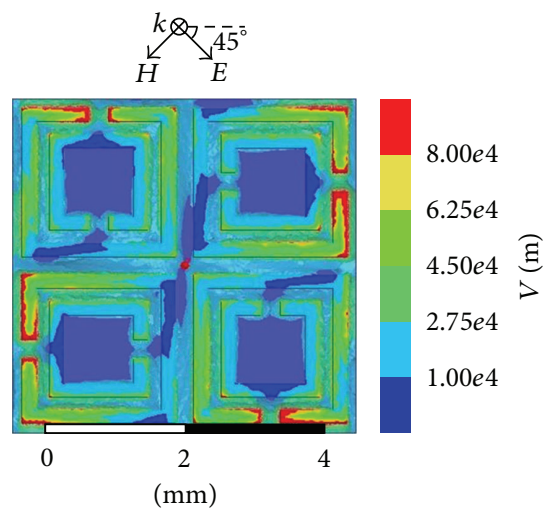

(c)

FIGURE 5: Electric field distribution at $10.27 \mathrm{GHz}$ for (a) normal incident TE, (b) normal incident TM, and (c) $45^{\circ}$ incident waves.

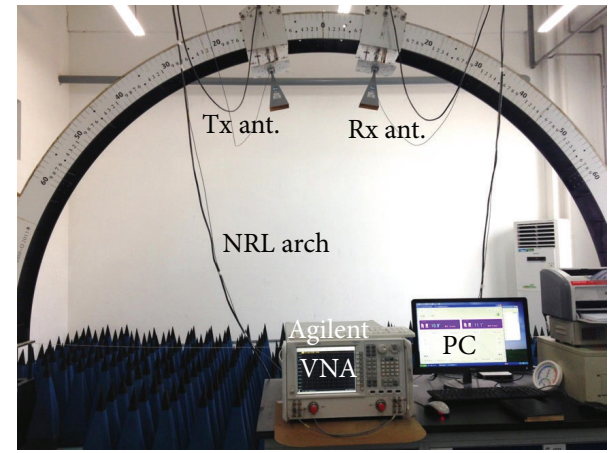

(a)

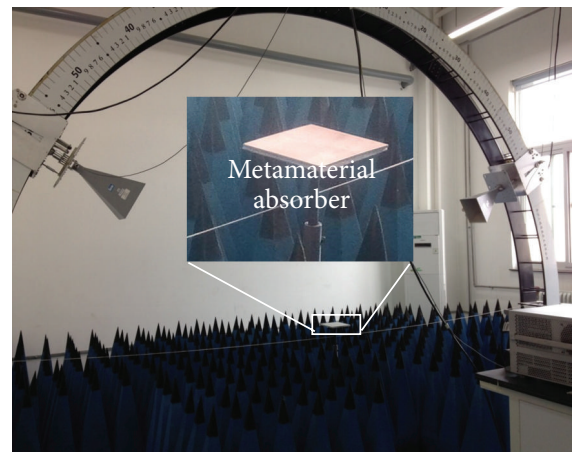

(b)

FIGURE 6: NRL arch used to measure the absorption of the MA at different incident angles. (a) Front and (b) side view of the experimental setup. The insert in (b) shows the zoomed view of the MA under measurement.

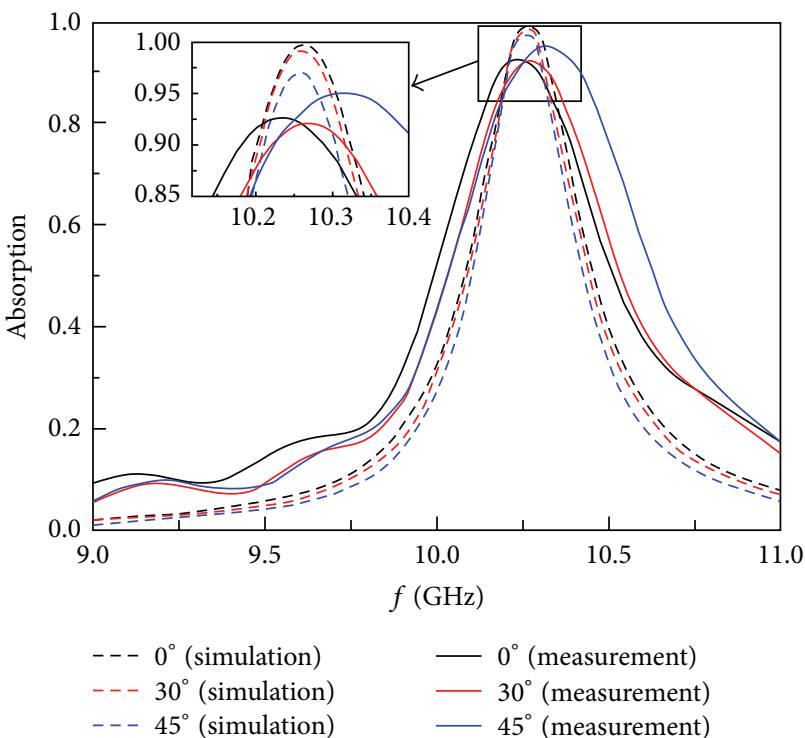

Figure 7: Simulated and measured absorptions at different angles $(0,30$, and 45 degrees). of $95.2 \%$ at $10.3 \mathrm{GHz}$, as shown in Figure 7. It is worth noting that the difference between the simulations and measurements can be attributed to the fabrication tolerance and measurement calibration.

The measured FBW of the MA is about $5 \%$ (FWHM $\cong$ $0.5 \mathrm{GHz}, f_{c} \cong 10.3 \mathrm{GHz}$ ), which is good enough for many practical applications. For example, both the microstrip patch antenna and the waveguide slot antenna generally need less than $3 \%$ FBW for efficient operations $[35,36]$. Hence, this MA is a promising candidate for radar cross section reduction of antennas and antenna arrays.

\section{Conclusions}

The theoretical analysis, full-wave FEM simulation, fabrication, and measurement of an X-band polarizationindependent and angle-insensitive MA are demonstrated. The MA employs 90-degree-rotated SRRs as a unit cell, which shows the absorption FBW of 5\% with incident angles ranging from 0 to 45 degrees by experiment. The measured results are in good agreement with the theoretical analysis 
and the full-wave FEM simulated ones, which provide effectively design guidelines for MAs. The proposed MA exhibits simple planar structure, easy fabrication using PCB technology, polarization-independence, angle-insensitiveness, and sufficient effective band. These remarkable features allow the MA to be a promising candidate for future applications, such as bolometer, perfectly matched layer, and scattering reductions.

\section{Conflict of Interests}

The authors declare that there is no conflict of interests regarding the publication of this paper.

\section{Acknowledgments}

This work was supported by the National Natural Science Foundation of China (Grants nos. 61307128, 61401025, 61371044, and 61275107), the Specialized Research Fund for the Doctoral Program of Higher Education of China (Grant no. 20131101120027), the Open Research Fund of The Academy of Satellite Application (Grant no. 2014_CXJJTX_03), the Beijing Natural Science Foundation (Grant no. 4154085), the Shanghai Academy of Spaceflight Technology (Grant no. SAST_201439), and the Basic Research Foundation of Beijing Institute of Technology, China (Grants nos. 20120542015, 20130542010, 20130542016, and 20140542002).

\section{References}

[1] V. G. Veselago, "The electrodynamics of substances with simultaneously negative values of $\varepsilon$ and $\mu$," Soviet Physics Uspekhi, vol. 10, no. 4, pp. 509-514, 1968.

[2] J. B. Pendry, A. J. Holden, D. J. Robbins, and W. J. Stewart, "Magnetism from conductors and enhanced nonlinear phenomena," IEEE Transactions on Microwave Theory and Techniques, vol. 47, no. 11, pp. 2075-2084, 1999.

[3] D. H. Werner and D. H. Kwon, Transformation Electromagnetics and Metamaterials: Fundamental Principles and Applications, Springer, London, UK, 2014.

[4] G. V. Eleftheriades and N. Engheta, "Metamaterials: fundamentals and applications in the microwave and optical regimes," Proceedings of the IEEE, vol. 99, no. 10, pp. 1618-1621, 2011.

[5] N. Engheta and R. W. Ziolkowski, Metamaterials: Physics and Engineering Explorations, John Wiley \& Sons, New York, NY, USA, 2006.

[6] B. Liu and C. L. Ji, "Bayesian nonparametric modeling for rapid design of metamaterial microstructures," International Journal of Antennas and Propagation, vol. 2014, Article ID 165102, 9 pages, 2014.

[7] J. Li and J. B. Pendry, "Hiding under the carpet: a new strategy for cloaking," Physical Review Letters, vol. 101, no. 20, Article ID 203901, 2008.

[8] Q. Wu, K. Zhang, F.-Y. Meng, and L.-W. Li, "Material parameters characterization for arbitrary $\mathrm{N}$-sided regular polygonal invisible cloak," Journal of Physics D: Applied Physics, vol. 42, no. 3, Article ID 035408, 2009.

[9] J. B. Pendry, "Negative refraction makes a perfect lens," Physical Review Letters, vol. 85, no. 18, pp. 3966-3969, 2000.
[10] T. J. Cui, D. R. Smith, and R. P. Liu, Metamaterials: Theory, Design and Applications, Springer, Berlin, Germany, 2009.

[11] W. R. Zhu, I. D. Rukhlenko, and M. Premaratne, "Graphene metamaterial for optical reflection modulation," Applied Physics Letters, vol. 102, no. 24, Article ID 241914, 2013.

[12] Q.-L. Zhang, L.-M. Si, Y. Huang, X. Lv, and W. Zhu, "Lowindex-metamaterial for gain enhancement of planar terahertz antenna," AIP Advances, vol. 4, no. 3, Article ID 037103, 2014.

[13] W. R. Zhu, I. D. Rukhlenko, F. Xiao, and M. Premaratne, "Polarization conversion in U-shaped chiral metamaterial with four-fold symmetry breaking," Journal of Applied Physics, vol. 115, no. 14, Article ID 143101, 2014.

[14] L. M. Si, Q. L. Zhang, W. D. Hu et al., "A uniplanar triple-band dipole antenna using complementary capacitively loaded loop," IEEE Antennas and Wireless Propagation Letters, vol. 14, pp. 743746, 2015.

[15] N. I. Landy, S. Sajuyigbe, J. J. Mock, D. R. Smith, and W. J. Padilla, "Perfect metamaterial absorber," Physical Review Letters, vol. 100, no. 20, Article ID 207402, 2008.

[16] J. B. Sun, L. Y. Liu, G. Y. Dong, and J. Zhou, "An extremely broad band metamaterial absorber based on destructive interference," Optics Express, vol. 19, no. 22, pp. 21155-21162, 2011.

[17] Y.-J. Huang, G.-J. Wen, J. Li, W.-R. Zhu, P. Wang, and Y.-H. Sun, "Wide-angle and polarization-independent metamaterial absorber based on snowflake-shaped configuration," Journal of Electromagnetic Waves and Applications, vol. 27, no. 5, pp. 552559, 2013.

[18] G. H. Yang, X. X. Liu, Y. L. Lv, J. H. Fu, Q. Wu, and X. M. $\mathrm{Gu}$, "An extremely broad band metamaterial absorber based on destructive interference," Journal of Applied Physics, vol. 115, Article ID 17E523, 2014.

[19] Y. J. Huang, G. J. Wen, W. R. Zhu, J. Li, L. M. Si, and M. Premaratne, "Experimental demonstration of a magnetically tunable ferrite based metamaterial absorber," Optics Express, vol. 22, no. 13, pp. 16408-16417, 2014.

[20] X. L. Liu, T. Starr, A. F. Starr, and W. J. Padilla, "Infrared spatial and frequency selective metamaterial with near-unity absorbance," Physical Review Letters, vol. 104, Article ID 207403, 2010.

[21] W. R. Zhu, X. P. Zhao, B. Gong, L. Liu, and B. Su, "Optical metamaterial absorber based on leaf-shaped cells," Applied Physics A, vol. 102, no. 1, pp. 147-151, 2011.

[22] B. Wang, T. Koschny, and C. M. Soukoulis, "Wide-angle and polarization-independent chiral metamaterial absorber," Physical Review B, vol. 80, no. 3, Article ID 033108, 2009.

[23] B. Zhu, Z. Wang, C. Huang, Y. Feng, J. Zhao, and T. Jiang, "Polarization insensitive metamaterial absorber with wide incident angle," Progress in Electromagnetics Research, vol. 101, pp. 231239, 2010.

[24] A. Dimitriadis, N. Kantartzis, and T. Tsiboukis, "A polarization/angle-insensitive, bandwidth-optimized, metamaterial absorber in the microwave regime," Applied Physics A, vol. 109, no. 4, pp. 1065-1070, 2012.

[25] K. B. Alici, A. B. Turhan, C. M. Soukoulis, and E. Ozbay, "Optically thin composite resonant absorber at the near-infrared band: a polarization independent and spectrally broadband configuration," Optics Express, vol. 19, no. 15, pp. 14260-14267, 2011.

[26] X. Shen, T. J. Cui, J. Zhao, H. F. Ma, W. X. Jiang, and H. Li, "Polarization-independent wide-angle triple-band metamaterial absorber," Optics Express, vol. 19, no. 10, pp. 9401-9407, 2011. 
[27] F. Dincer, M. Karaaslan, E. Unal, K. Delihacioglu, and C. Sabah, "Design of polarization and incident angle insensitive dual-band metamaterial absorber based on isotropic resonator," Progress in Electromagnetics Research, vol. 144, pp. 123-132, 2014.

[28] M. R. Soheilifar, R. A. Sadeghzadeh, and H. Gobadi, "Design and fabrication of a metamaterial absorber in the microwave range," Microwave and Optical Technology Letters, vol. 56, no. 8, pp. 1748-1752, 2014.

[29] M. Soheilifar and R. Sadeghzadeh, "Design, simulation, and fabrication of an ultrathin planar microwave metamaterial absorber," Microwave and Optical Technology Letters, vol. 56, no. 12, pp. 2916-2922, 2014.

[30] H. Tao, C. M. Bingham, A. C. Strikwerda et al., "Highly flexible wide angle of incidence terahertz metamaterial absorber: design, fabrication, and characterization," Physical Review B, vol. 78, no. 24, Article ID 241103, 2008.

[31] D. R. Smith, S. Schultz, P. Markoš, and C. M. Soukoulis, "Determination of effective permittivity and permeability of metamaterials from reflection and transmission coefficients," Physical Review B, vol. 65, no. 19, Article ID 195104, 2002.

[32] D. R. Smith, D. C. Vier, T. Koschny, and C. M. Soukoulis, "Electromagnetic parameter retrieval from inhomogeneous metamaterials," Physical Review E-Statistical, Nonlinear, and Soft Matter Physics, vol. 71, no. 3, Article ID 036617, 2005.

[33] X. Chen, T. M. Grzegorczyk, B.-I. Wu, J. Pacheco Jr., and J. A. Kong, "Robust method to retrieve the constitutive effective parameters of metamaterials," Physical Review E, vol. 70, no. 1, Article ID 016608, 2004.

[34] R. Faez, I. M. Martin, M.-A. de Paoli, and M. C. Rezende, "Influence of processing time and composition in the microwave absorption of EPDM/PAni blends," Journal of Applied Polymer Science, vol. 83, no. 7, pp. 1568-1575, 2002.

[35] L.-M. Si, Y. Liu, Y. Huang, and W. Zhu, "Ka-band slotmicrostrip-covered and waveguide-cavity-backed monopulse antenna array," International Journal of Antennas and Propagation, vol. 2014, Article ID 707491, 5 pages, 2014.

[36] T. Liu, X. Y. Cao, J. Gao, Q. R. Zheng, W. Q. Li, and H. H. Yang, "RCS reduction of waveguide slot antenna with metamaterial absorber," IEEE Transactions on Antennas and Propagation, vol. 61, no. 3, pp. 1479-1484, 2013. 

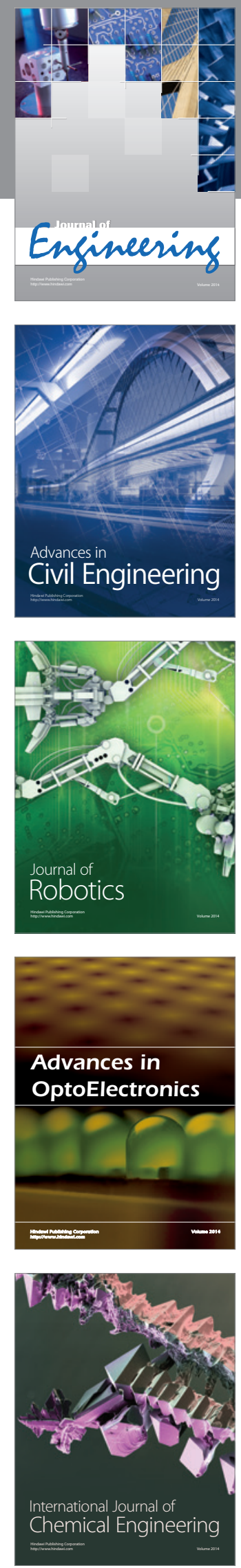

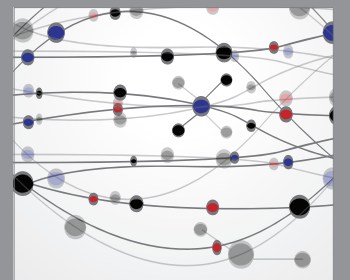

The Scientific World Journal
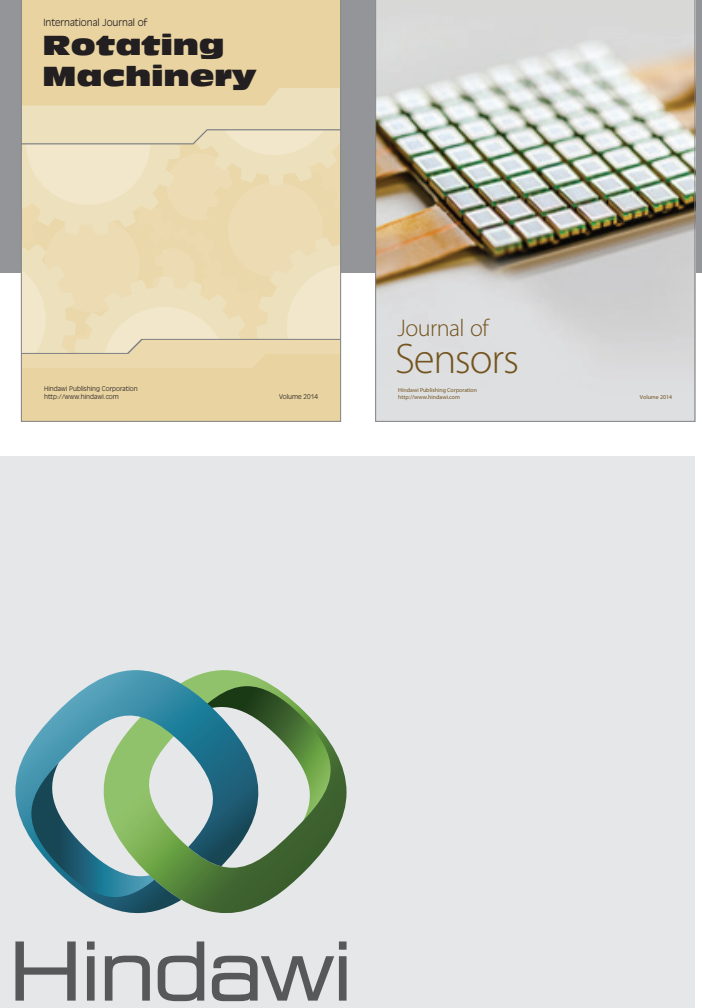

Submit your manuscripts at http://www.hindawi.com
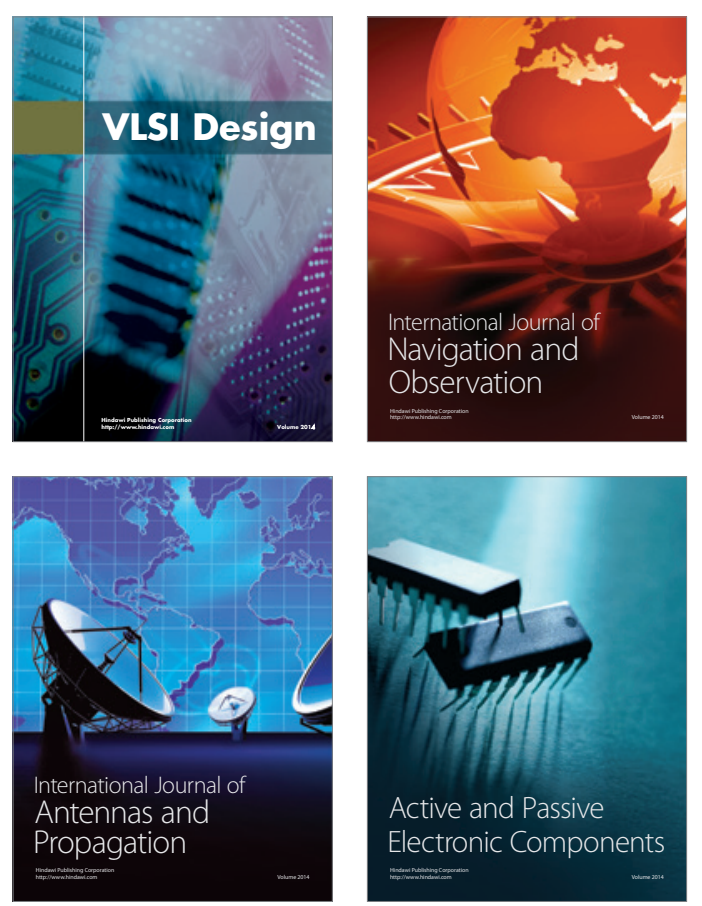
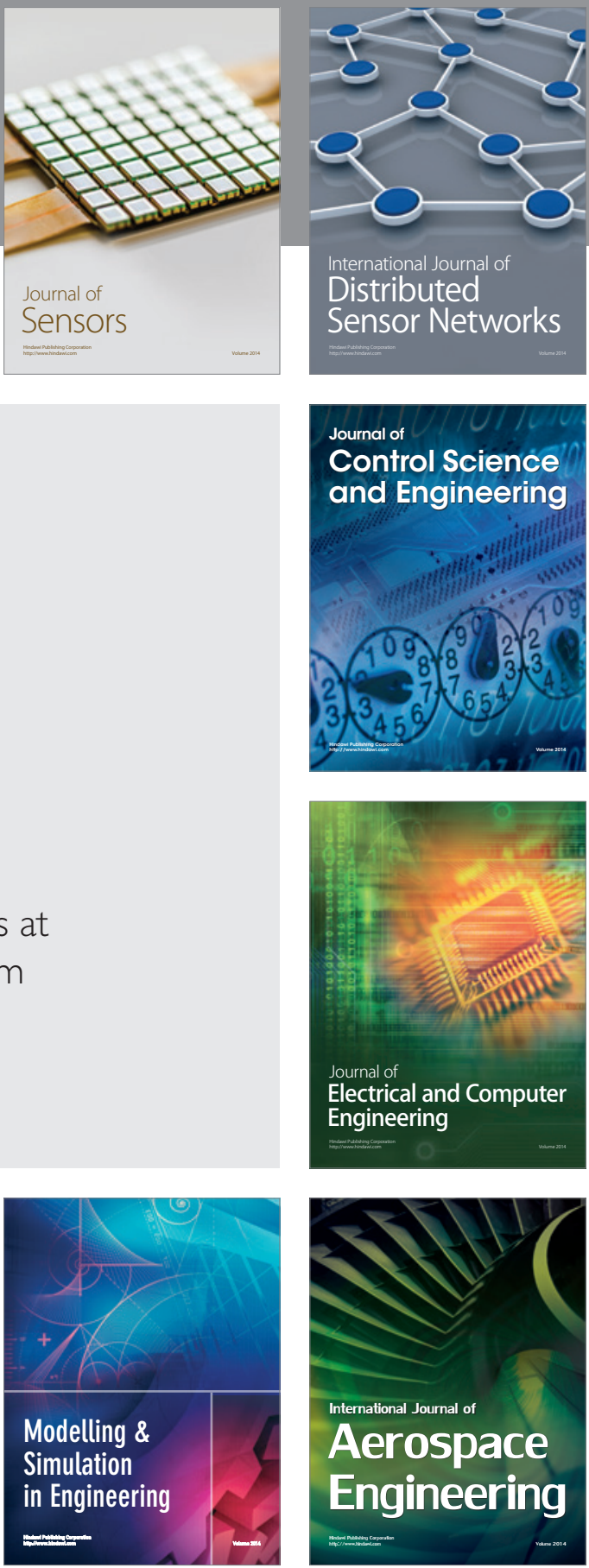

Journal of

Control Science

and Engineering
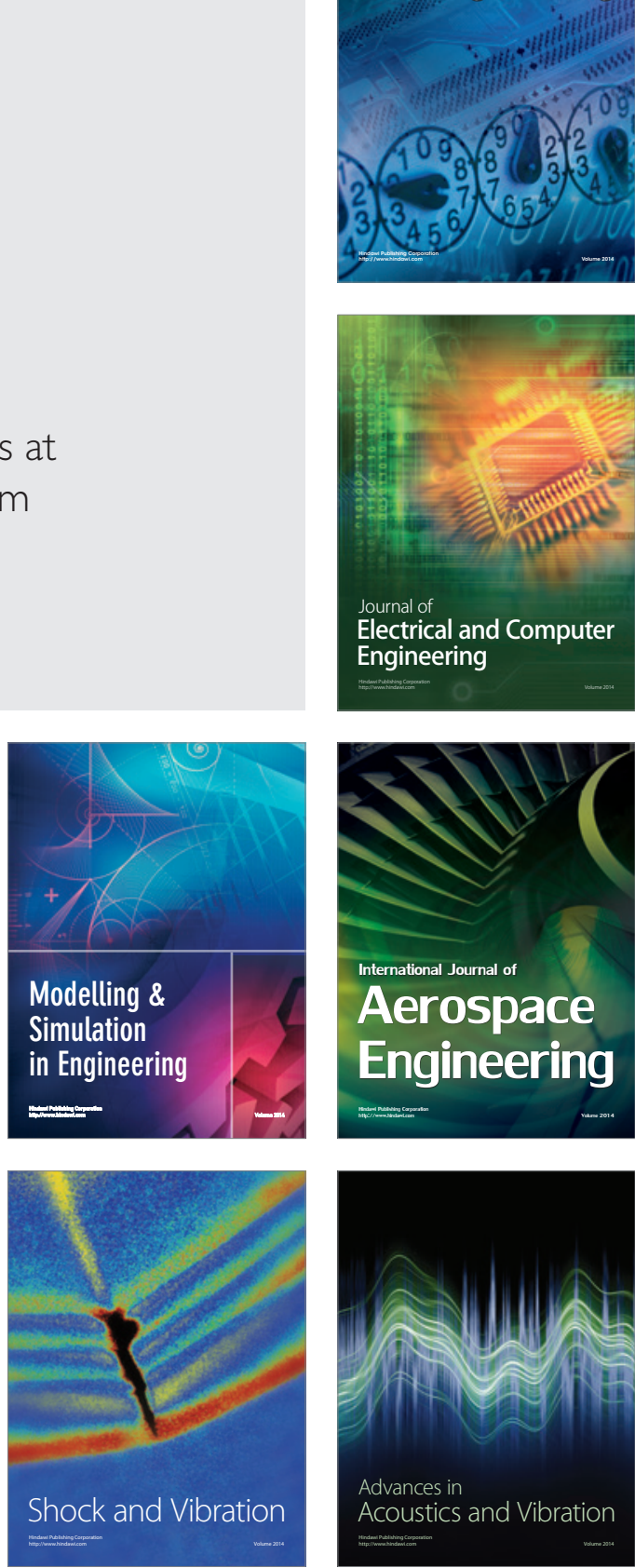